\title{
Liga Acadêmica de Fisiologia Médica: um olhar dos discentes sobre metodologias ativas de ensino-aprendizagem
}

\author{
Academic League of Medical Physiology: a view of the students about active teaching-learning \\ methodologies
}

Rafael Augusto Frizzo ${ }^{1 *}$, Marina Carla Kornowski ${ }^{1}$, Isabel Oliveira de Oliveira', Gustavo Dias Ferreira ${ }^{1}$

${ }^{1}$ Universidade Federal de Pelotas

*e-mail: r-frizzo@hotmail.com

\begin{abstract}
Resumo
A Liga Acadêmica de Fisiologia Médica (LAFIM) oportuniza o estudo aplicado da fisiologia básica à prática clínica. O objetivo foi avaliar a influência da LAFIM na formação dos integrantes do projeto e a visão dos monitorados acerca das ações realizadas durante o semestre para a melhoria do processo de aprendizagem. Foram aplicados dois questionários, um para os alunos participantes da Liga e outro para os acadêmicos que cursaram as disciplinas de Fisiologia do primeiro e do segundo semestre do Curso de Medicina, nas quais a intervenção das atividades desenvolvidas pelo projeto foi aplicada. Os participantes do projeto mostraram satisfação com os encontros e discussões da Liga, além de relatarem que a experiência de "ser monitor" foi positiva para suas formações acadêmicas. Em relação aos monitorados, foi observada uma diferença na percepção e envolvimento quanto aos casos clínicos, sendo que os alunos do segundo semestre apresentam um maior aproveitamento da atividade quando comparados aos ingressantes, em contrapartida, os acadêmicos do primeiro semestre relataram melhor aproveitamento das monitorias. Foi possível concluir que a Liga é um método de estudo na área de fisiologia que pode complementar o ensino tanto dos participantes do projeto, como dos discentes que estão cursando a disciplina.
\end{abstract}

Palavras-chave: Fisiologia; Ensino; Medicina.

\begin{abstract}
The Academic League of Medical Physiology provides an applied study from basic physiology to clinical practice. The objective was to evaluate the influence of the League in the formation of the project members and the view of the monitored students about the activities for their learning. Two questionnaires were applied, one for the students participating in the League and another for the first and second semester students of the Medicine Course who attended Physiology class and suffered an intervention through the activities developed by project. Project participants reported satisfaction with the meetings and discussions of the League, and that the experience like monitor had added to their academic backgrounds. In relation to the monitored students, it was noticed that there was a difference in the perception and use of the clinical cases, and the students of the second have a greater use of the activity, on the other hand, the first semester students reported better use of the monitoring. It was concluded that the League is a method in the field of Physiology that can complement the teaching of both groups of students, the project participants and the students who are studying the discipline.
\end{abstract}

Keywords: Physiology; Teaching; Medicine. 
Pesquisa em ensino: Academic League of Medical Physiology: a view of the students about active teachinglearning methodologies

\section{Introdução}

Um dos desafios recorrentes no Ensino Superior é utilizar estratégias para minimizar a evasão e possibilitar que os acadêmicos sejam críticos e desenvolvam competências e habilidades demandadas pelo mundo contemporâneo [1]. É papel do Ensino Superior não só adicionar conhecimentos científicos (teóricos e práticos), mas também proporcionar a aprendizagem como um processo ativo, cognitivo e construtivo, imbricada com sua função social [2].

Os ingressantes das faculdades de medicina possuem uma grande diversidade na educação escolar, portanto, torna-se um desafio para os docentes o ensino uniforme da disciplina. Uma maneira de atender a essas diferenças é estimular o uso de métodos de ensino-aprendizagem com os quais os discentes melhor se adaptam [3]. Das muitas técnicas pedagógicas utilizadas no ensino superior, o modelo expositivo é o mais utilizado nos anos iniciais. No entanto, a geração atual de discentes tem características que podem favorecer os processos de aprendizagem ativa, nos quais os alunos se envolvem cognitivamente com o material de várias maneiras, resultando em melhor compreensão e retenção $[4,5]$.

As metodologias ativas de ensino-aprendizagem são um modelo no qual os discentes são mais participantes e engajados na aula, com utilização de exercícios, jogos, problemas, debates e discussões de casos clínicos, por exemplo [6]. Além disso, a abordagem colaborativa de ensino, na qual os estudantes interagem com o professor e entre si, permitem uma compreensão mais eficaz do conteúdo, o que melhora a capacidade de retenção do conhecimento da fisiologia humana [7].

Estudos têm demonstrado melhor performance acadêmica quando os estudantes são retirados da posição de meros espectadores e se tornam responsáveis pela organização e apresentação das atividades de ensino. Além disso, o uso de estratégias colaborativas e metodologias ativas permite um melhor entendimento de conteúdos complexos, proporcionando aquisição de diferentes competências profissionais [8, 9]. A superação de uma abordagem tradicional de ensino deve ser estimulada, buscando promover uma aprendizagem ativa, que parta de questões concretas da realidade de atuação do acadêmico, e possibilite articular os conhecimentos teóricos às situações reais [10].

Nesse sentido, o professor passa a exercer um papel de mediador da aprendizagem, despertando a curiosidade científica e o prazer em obter conhecimento [11]. Tal mediação vai para além da sala de aula e do currículo tradicional, existindo 
Pesquisa em ensino: Academic League of Medical Physiology: a view of the students about active teachinglearning methodologies

também em atividades extracurriculares, que são ferramentas que melhoram a motivação dos discentes e criam conexões com a futura prática profissional [12, 13].

Buscar metodologias de ensino que facilitem o processo ensino-aprendizagem é uma função docente a ser exercida rotineiramente, considerando a diversidade e especificidade dos discentes [14]. Uma dessas estratégias diversificadas de ensino que é muito comum entre os cursos de Medicina é a criação de Ligas Acadêmicas (LA), formadas por grupos de alunos de diferentes anos da graduação e professores vinculados à Instituição [15]. Esses grupos promovem conhecimento e atuação em áreas específicas, sendo regidas por três princípios básicos: atividades de pesquisa, ensino e assistência [16].

A disciplina de fisiologia é um componente da base curricular obrigatória dos cursos de graduação na área da saúde [17]. Tal obrigatoriedade se dá em virtude da inexorável necessidade do entendimento do funcionamento do corpo humano para, em seguida, o aluno ser capaz de compreender a natureza dos processos fisiopatológicos e farmacológicos, com o propósito de proporcionar maior efetividade na prática clínica e terapêutica [18]. No entanto, os currículos dos cursos de graduação nem sempre são suficientes para o completo preparo acadêmico, dessa forma, os discentes que buscam atividades adicionais, como as LAs, podem aproveitar mais o período de formação [19].

Nesse contexto, o projeto denominado Liga Acadêmica de Fisiologia Médica (LAFIM) foi criado com objetivo de expandir o ensino nas disciplinas de Fisiologia I e II no Curso de Medicina, vinculado ao Departamento de Fisiologia e Farmacologia, do Instituto de Biologia da Universidade Federal de Pelotas. Como as LAs são extracurriculares, professores e alunos participantes aprofundam os conteúdos das disciplinas, possibilitando a construção de novos conhecimentos e vivências, com maior flexibilização curricular, protagonismo estudantil, buscando um espaço de pertencimento e de autenticidade [20, 21].

Os alunos que cursam as disciplinas de fisiologia pertencem ao primeiro ano do Curso (primeiro e segundo semestres). A estratégia utilizada no projeto foi a apresentação de casos clínicos para os ingressantes, atividade realizada pelos participantes da Liga, os quais são acadêmicos de semestres mais avançados. Dessa forma, era esperada a aplicação do conhecimento básico, reforçando o conteúdo exposto pelo professor da disciplina, somado ao conhecimento adquirido pelos acadêmicos nas atividades teóricas e práticas vivenciadas com o avançar do curso. Por meio de debates e discussões, foi almejado aprimorar, consolidar e atualizar a aplicação da fisiologia humana, além de 
Pesquisa em ensino: Academic League of Medical Physiology: a view of the students about active teachinglearning methodologies

instigar a aprendizagem por meio da leitura de artigos científicos que associem a fisiologia básica à prática médica.

Além disso, os acadêmicos da Liga, que já haviam cursado a disciplina, também deveriam ministrar monitorias. As tarefas assumidas pelos alunos monitores tiveram como objetivo reforçar a compreensão sobre o conteúdo abordado, a fim de tirar dúvidas dos alunos, e ajudar no combate à reprovação e a evasão. Uma vez que o aluno monitor já conhece o conteúdo a ser ministrado, a didática do professor, e normalmente tem proximidade com os alunos da disciplina, ele participa ativamente como um facilitador do processo de ensino e aprendizagem, esclarecendo dúvidas em horários alternativos ou ajudando na sala de aula. Trata-se, portanto, de uma prática que possibilita o desenvolvimento de experiência pedagógica que fortalece o compartilhamento de conhecimentos e a transformação do aluno em sujeito ativo do processo de ensinoaprendizagem [22].

Com isso, o objetivo deste trabalho foi avaliar as potencialidades e limitações da LAFIM na formação dos acadêmicos integrantes do projeto e a visão dos monitorados acerca do impacto das atividades desenvolvidas, uma vez que a percepção discente sobre o uso de metodologias ativas podem colaborar com o aprimoramento do ensino em fisiologia [9].

\subsection{Um pouco mais sobre o projeto}

LAFIM é um projeto de ensino criado no ano de 2014 vinculado ao Instituto de Biologia da Universidade Federal de Pelotas. No momento da realização deste trabalho contava com a participação de 14 acadêmicos (do terceiro ao sétimo semestre) do Curso de Medicina, sendo dois bolsistas de monitoria, um bolsista de ensino e 11 monitores voluntários, além de quatro docentes da Instituição. Para ser membro da LAFIM o acadêmico deveria ter concluído as duas disciplinas curriculares de fisiologia humana, demonstrar interesse e passar por processo específico de seleção que se baseia em trajetória acadêmica, apresentação oral e entrevista.

$\mathrm{O}$ projeto foi organizado em três grupos de atividades principais. O primeiro correspondia a realização de reuniões quinzenais, nas quais os membros participantes apresentavam seminários a partir de artigos científicos que correlacionavam o conhecimento da fisiologia com o da patologia, da farmacologia e da clínica médica. $\mathrm{O}$ artigo era enviado previamente para que os demais integrantes do projeto pudessem ler e 
Pesquisa em ensino: Academic League of Medical Physiology: a view of the students about active teachinglearning methodologies

contribuir com a discussão. Essa atividade era supervisionada pelos professores que faziam parte do projeto.

O segundo grupo de trabalho desenvolvido pelos participantes da LAFIM dizia respeito às atividades de monitoria nas disciplinas de Fisiologia I e II para os acadêmicos dos dois primeiros semestres do curso de Medicina. Os monitores eram divididos de maneira ordenada e proporcional entre os dois semestres, disponibilizando assim, atividades de monitoria durante todo o período letivo.

Como terceira ação, os membros da Liga também apresentavam casos clínicos aplicados em aulas de discussão na disciplina de fisiologia em curso. O objetivo era associar o conteúdo do ciclo básico com as futuras disciplinas do currículo e incentivar os acadêmicos a ter um estudo problematizado, crítico e continuado.

\section{Métodos}

Foi realizado um estudo transversal descritivo, a partir de questionários próprios autoaplicáveis, para avaliar a influência da LAFIM na vida acadêmica dos estudantes de Medicina, divididos em dois eixos: um dos questionários foi aplicado aos acadêmicos participantes do projeto e o outro, aos acadêmicos que cursaram as disciplinas de Fisiologia I e II no primeiro semestre do ano letivo de 2017.

O questionário para todos os 14 acadêmicos participantes da LAFIM foi estruturado com 13 questões objetivas e 4 subjetivas. Os integrantes avaliaram separadamente as reuniões quinzenais com seminários e a dinâmica das monitorias, atribuindo conceitos (AInsuficiente; B- Regular; C- Bom; D- Muito Bom; E- Excelente) de acordo com uma escala Likert [9, 23, 24, 25].

A escala Likert baseia-se em uma série de afirmações para as quais são emitidos graus de concordância. Embora tenha sido desenvolvida em 1932, ainda é amplamente utilizada em pesquisas das mais diversas áreas do conhecimento, sendo que uma de suas vantagens é o seu fácil manuseio e compreensão.

Originalmente foi desenvolvida para ser aplicada com cinco pontos, em um espectro de total concordância à discordância completa. Com o seu uso frequente, foram desenvolvidos modelos com outras variações, a critério do pesquisador, com modificações das denominações da graduação, bem como do número de pontos [9, 23, $24,25]$. O presente estudo utilizou a escala de acordo com o exemplo do Quadro 1. 
Pesquisa em ensino: Academic League of Medical Physiology: a view of the students about active teachinglearning methodologies

Quadro 1. Exemplo escala Likert

\begin{tabular}{|c|c|c|c|c|}
\hline \multicolumn{5}{|c|}{ Você considera adequada a carga horária disponibilizada para os seminários/ } \\
discussão de artigos? \\
\hline Insuficiente & Regular & Bom & Muito Bom & Excelente \\
\hline 1 & 2 & 3 & 4 & 5 \\
\hline
\end{tabular}

Avaliaram-se, em relação às reuniões, os aspectos: carga horária, revisão dos conhecimentos, relação com a vivência na Faculdade de Medicina, motivação, sensação de aproveitamento, desenvolvimento das habilidades de comunicação, leitura de artigos e compartilhamento de conhecimentos. Já em relação às atividades de monitorias foram avaliados a sua influência para: correlacionar com outras disciplinas do curso, habilidades de comunicação, motivação, aproveitamento da monitoria para o seu conhecimento e carga horária das atividades. As questões subjetivas, por sua vez, abordavam os principais pontos positivos e negativos vivenciados pelos acadêmicos.

O outro questionário, aplicado aos alunos que cursavam as disciplinas de Fisiologia I e II, avaliou os aspectos gerais sobre a disciplina, as atividades de monitorias e os casos clínicos apresentados pelos monitores. A primeira parte do questionário foi utilizada para identificar a percepção dos alunos sobre: 1) desempenho na disciplina; 2) participação das atividades de casos clínicos e impacto no processo de ensino-aprendizado; 3) motivação para participar nas atividades de monitoria; 4) carga horária disponibilizada pelos monitores para as atividades de monitoria; 5) importância da disciplina para a prática médica futura. Cada sentença foi avaliada também e de acordo com a escala Likert : A) Insuficiente; B) Regular; C) Bom; D) Muito Bom; E) Excelente [9, 23, 24, 25].

Utilizou-se na segunda parte do questionário a mesma escala de avaliação, no entanto, as questões se referiram especificamente às atividades de monitoria, e, portanto, apenas os alunos que participaram ao menos uma vez das monitorias deveriam respondê-las. Foi avaliada a percepção dos acadêmicos aos seguintes pontos: 1) Influência das monitorias no desempenho acadêmico 2) Influência das monitorias na compreensão da matéria; 3) Clareza dos monitores para explicar a matéria; 4) Preparo e conhecimento dos monitores sobre os conteúdos ministrados. Na terceira e última parte do questionário, foram perguntados os motivos pelos quais os alunos não participaram das atividades de monitoria, através de uma questão de múltipla escolha englobando: “1) Falta de tempo; 2) Falta de interesse; 3) Indisponibilidade de horários; 4) Aprende mais estudando sozinho; 5) Outro. Qual?". 
Pesquisa em ensino: Academic League of Medical Physiology: a view of the students about active teachinglearning methodologies

Organizaram-se os dados em uma planilha do MS Excel 2013 e apresentados em frequência absoluta e relativa. Tal metodologia foi desenvolvida pelos autores, com base em trabalhos que avaliaram o ensino da fisiologia por meio de metodologias ativas e colaborativas $[3,4,5,7,9,26]$. O objetivo secundário foi colaborar com o desenvolvimento de metodologias alternativas, uma vez que as pesquisas de ensino em fisiologia necessitam de mais reconhecimento e apoio no Brasil [27].

\section{Resultados e Discussão}

Os resultados foram divididos em dois eixos, sendo o primeiro para avaliar a percepção dos acadêmicos integrantes da LAFIM e o segundo para analisar a visão dos acadêmicos monitorados.

\subsection{Avaliação dos integrantes do projeto}

A figura 1 mostra os resultados do primeiro eixo. Os 14 membros discentes do projeto responderam ao questionário. Em relação as reuniões quinzenais, obteve-se um predomínio do conceito "Excelente" (57\%). O aspecto mais bem avaliado foi o desenvolvimento de habilidades de apresentação oral, seguido pela importância para a prática médica futura e a carga horária do projeto. Já os que receberam avaliações piores, ainda que com predominância da categoria "Muito Bom", foram: aproveitamento e motivação na participação das discussões.

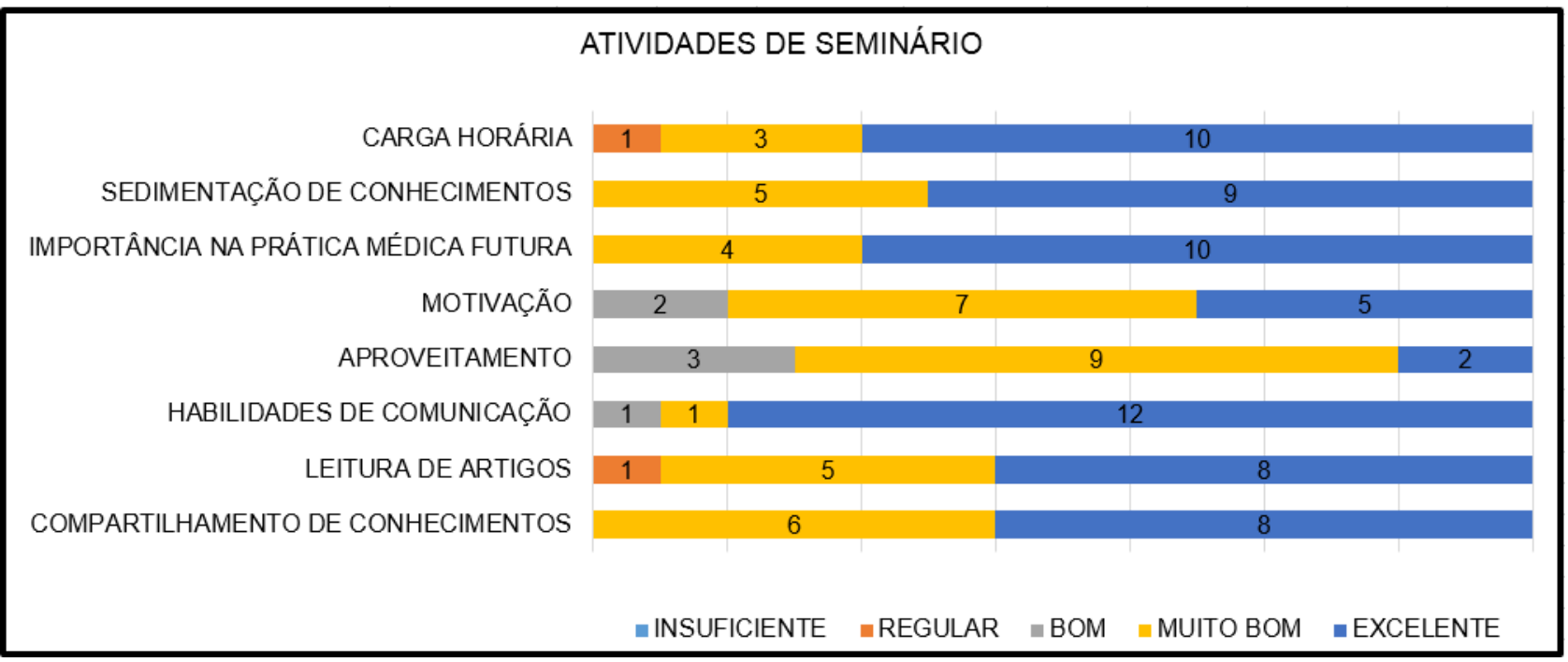

Figura 1. Percepção dos integrantes da LAFIM sobre as atividades de seminários, dados apresentados em frequência absoluta.

Esses resultados corroboraram com as respostas das questões subjetivas, onde foram destacados os pontos positivos e negativos das atividades quinzenais. 
Pesquisa em ensino: Academic League of Medical Physiology: a view of the students about active teachinglearning methodologies

"Nas discussões dos artigos temos a oportunidade de falar sobre assuntos mais voltados para nossa prática clínica associando a fisiologia a situações que vivenciaremos no cotidiano da nossa futura profissão; além disso, nos estimula a pensar nas aplicações da fisiologia e buscar entender assuntos que nem sempre vemos nas aulas habituais da faculdade." Acadêmico 1

Em relação aos pontos a serem melhorados nas atividades de encontro da LAFIM, a maioria dos alunos apontou a necessidade de haver mais discussões durante as apresentações:

"É necessário estimular mais a discussão entre os alunos, pois é o que realmente promove a formação do conhecimento. Também desenvolver uma análise crítica dos artigos trabalhados." Acadêmico 2

Em outro estudo, desenvolvido com acadêmicos da área da Farmácia e Fisioterapia na Universidade Federal do Pampa, também foi relatada a implementação de atividades fora da sala de aula, onde os alunos teriam que obter conhecimento por meio de um processo ativo. Eles creditaram o impacto positivo dessa outra metodologia ao fato de que a mesma permitiu que os alunos vivenciassem ações interdisciplinares, discussões e trocas de conhecimentos, corroborando com nossos resultados [8].

Já na segunda parte do questionário para os participantes do projeto, quanto à prática das monitorias, o resultado mais prevalente foi o conceito "Excelente" (76\%). Nesse quesito, os aspectos que receberam as melhores avaliações foram: desenvolvimento de habilidades de comunicação e correlação entre a atividade da monitoria em fisiologia com as demais disciplinas do curso. Por fim, motivação e carga horária receberam conceitos piores, mas, ainda assim, positivos. Um dos participantes do projeto não respondeu a esta parte do questionário, sendo, contabilizadas, portanto, a resposta de 13 participantes (Figura 2).

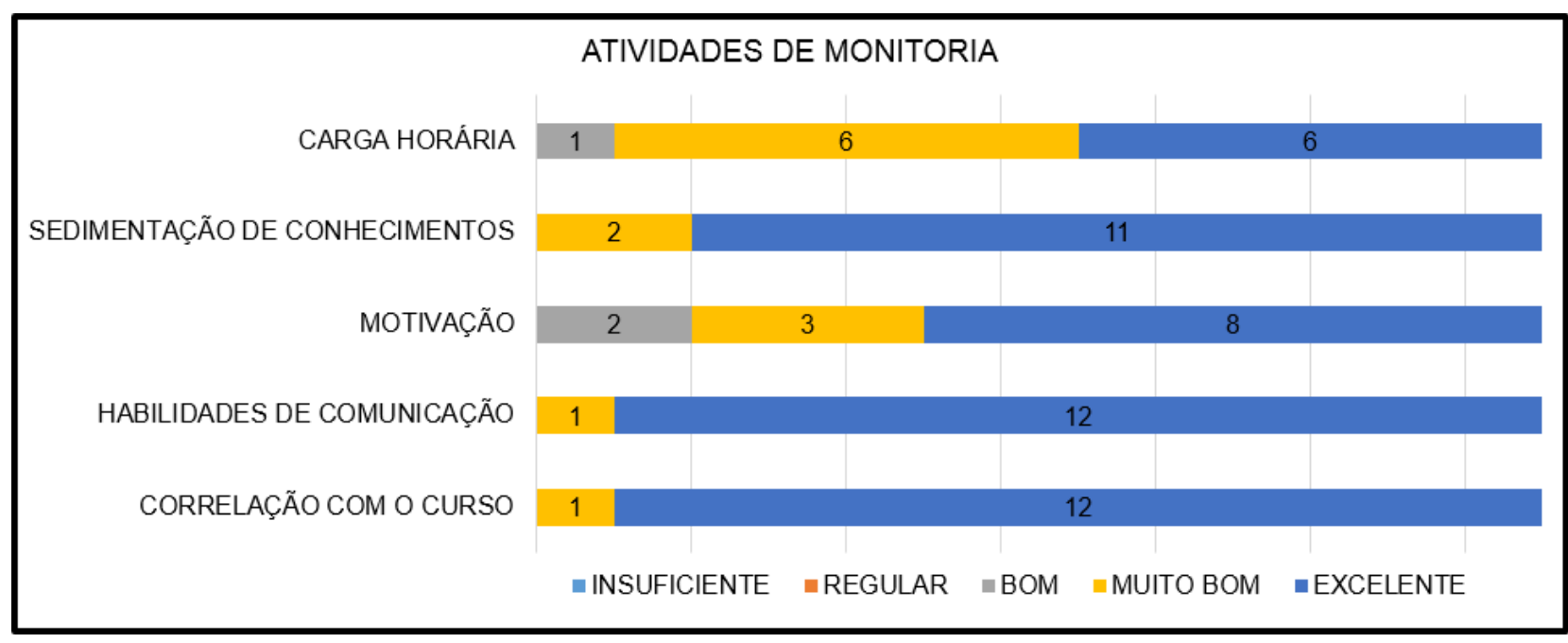

Figura 2. Percepção dos integrantes da LAFIM sobre as atividades de monitoria, dados apresentados em frequência absoluta. 
Outros estudos também mostram que a monitoria é uma modalidade que contribui para a formação integrada do acadêmico nas diversas atividades dos cursos de graduação, sendo um instrumento para a melhoria do ensino, por meio de novas experiências pedagógicas que fortalecem a articulação entre a teoria e a prática, além de promover a cooperação mútua entre discente e docente [22]. O aluno-monitor desenvolve habilidades intelectuais e sociais necessárias, dinamizando e contextualizando os conteúdos da disciplina que monitora, reconstruindo com os estudantes conhecimentos acerca dos assuntos abordados, ao mesmo tempo em que adquire experiências positivas para sua formação [28].

As respostas discursivas dessa parte do questionário também mostram pontos positivos como: oportunidade para revisão dos conteúdos; aprimoramento e aprofundamento do conhecimento; desenvolvimento de capacidade de síntese e comunicação; interação com alunos de diferentes semestres, o que proporciona um conhecimento compartilhado; entendimento da fisiologia como disciplina fundamental e útil durante todo o curso; e desenvolvimento de habilidades e aspiração à docência, em consonância com resultado de outros estudos que igualmente demonstram que, além do supracitado, a inserção do estudante em LAs possibilita o trabalho em equipe e maximiza a comunicação interpessoal [29].

Os principais aspectos a serem melhorados, em relação aos alunos monitorados, foram que esses poderiam ter uma maior interação, com participação mais ativa e dinâmica; com maior estudo prévio ao encontro a fim de haver mais questionamentos e fazer correlações clínicas. Outro apontamento por parte dos monitores foi a baixa adesão dos alunos em algumas ocasiões, podendo isso ter impactado de forma negativa na avaliação acerca da motivação. Foi constatada uma maior participação na atividade quando os encontros antecediam as provas.

Sugere-se que isso tenha ocorrido por alguns fatores como o hábito de procrastinação das tarefas acadêmicas, ou a dificuldade em encontrar um horário viável para monitores e monitorados, principalmente por estes serem de semestres diferentes e o curso apresentar uma carga horária alta. Em relação a esse aspecto, foram propostas soluções como a criação de um grupo nas redes sociais para aproximar monitores e alunos, e a busca ativa por alunos que apresentem maiores dificuldades, tentando assim diminuir os índices de reprovação na disciplina. 
Pesquisa em ensino: Academic League of Medical Physiology: a view of the students about active teachinglearning methodologies

\subsection{Avaliação dos acadêmicos que cursaram Fisiologia I e II}

O segundo eixo do estudo, responsável por avaliar a percepção dos acadêmicos beneficiados pelas atividades da Liga, detectou os seguintes resultados: dentre os alunos que responderam ao questionário, 45 cursaram a disciplina de Fisiologia I e 43 cursaram a disciplina de Fisiologia II no primeiro semestre letivo do ano de 2017. Cada turma é composta por 50 alunos, portanto, esta quantidade representa $86 \%$ e $90 \%$ do total, respectivamente. A maioria dos alunos concordou totalmente com a afirmativa de que 0 conhecimento da fisiologia é importante para a prática médica futura: 93,3\% dos que cursaram Fisiologia I e 95,3\% dos que cursaram Fisiologia II. Dessa forma, fica evidenciada a visão dos discentes sobre a importância do conhecimento da fisiologia em sua formação.

Levando em consideração que os monitores vinculados ao projeto de ensino também são acadêmicos do curso de medicina e, portanto, apresentam uma carga horária similar aos alunos monitorados, por vezes a disponibilidade de horários para as atividades de monitoria pode ser limitada. Essa condição, no entanto, foi contornada pela divisão dos conteúdos e horários entre os 14 monitores, fazendo com que as atividades pudessem ser garantidas da melhor forma possível. Em relação a esse quesito, $40 \%$ dos alunos que cursaram Fisiologia I e $46,5 \%$ dos alunos que cursaram Fisiologia II classificaram como "Bom" os horários disponibilizados pelos monitores.

Dos alunos que cursaram a disciplina de Fisiologia I, 42,2\% responderam que consideram "Muito bom" sua motivação para participar das atividades de monitoria. Diferentemente daqueles que cursaram Fisiologia II: apenas 23,25\% responderam desta forma este quesito. Os resultados mostram uma perda de interesse dos acadêmicos em participar das atividades de monitoria de um semestre para o outro, ou talvez tenha um fator de confusão, visto que o segundo semestre do curso tem uma carga horária mais densa, sobrando menos tempo para outras atividades. Aqui é possível perceber uma limitação do estudo: o questionário criado não conseguiu elucidar a assiduidade dos alunos monitorados com mais detalhamento. Não se sabe se eram sempre os mesmos que participavam da monitoria com maior frequência ou se havia uma variabilidade maior entre os estudantes. Esse fator será corrigido para as próximas análises, a fim de intervir da melhor forma possível.

Em relação ao desempenho dos alunos na disciplina, a maioria dos que cursaram Fisiologia I ( $51,1 \%$ ) classificou seu desempenho como "Bom", sendo que $40 \%$ dos alunos classificaram seu desempenho como "Muito Bom". Dentre os alunos que cursaram 
Pesquisa em ensino: Academic League of Medical Physiology: a view of the students about active teachinglearning methodologies

Fisiologia II, 44,1\% classificou seu desempenho como "Muito Bom" e 37,2\% como "Bom", 9\% classificou como Excelente, e apenas 6\% como "Regular" ou "Ruim". A comparação dos acadêmicos da Fisiologia I e Il podem ser visualizadas nas Figuras 3 e 4.

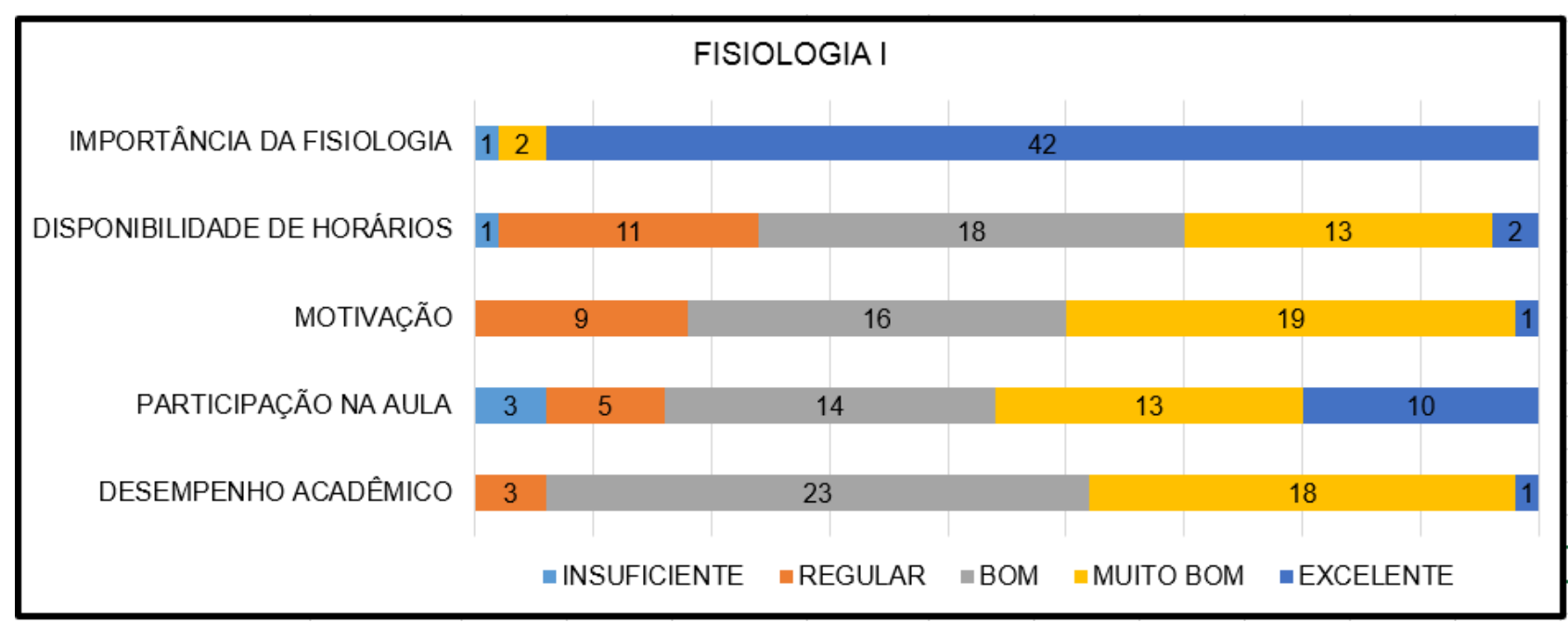

Figura 3. Percepção dos estudantes de Fisiologia I sobre aspectos gerais da disciplina e influência da LAFIM no primeiro semestre, dados apresentados em frequência absoluta.

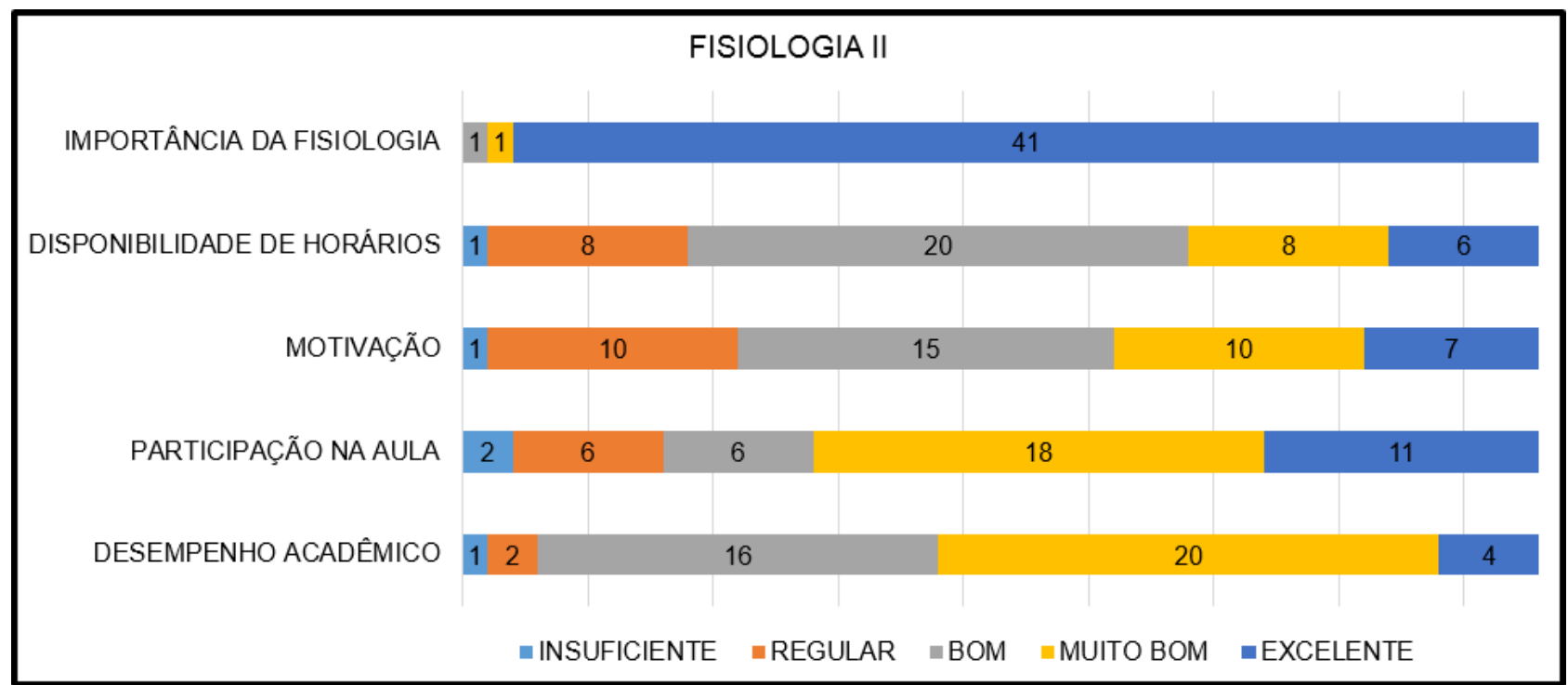

Figura 4. Percepção dos estudantes de Fisiologia II sobre aspectos gerais da disciplina e influência da LAFIM no segundo semestre, dados apresentados em frequência absoluta.

A segunda parte do questionário foi respondida apenas pelos alunos que participaram ao menos uma vez das atividades de monitoria, sendo que da Fisiologia I foram 68,8\% (31 alunos). Foram avaliados percepção de conhecimento teórico do monitor e transmissão de conteúdo (didática), além do impacto dessa atividade para o desempenho e compreensão do conteúdo dos estudantes na disciplina. Todos os quesitos evidenciaram uma percepção positiva (predominância de "Muito Bom" e "Excelente") dos alunos em relação às monitorias (Figura 5). 
Pesquisa em ensino: Academic League of Medical Physiology: a view of the students about active teachinglearning methodologies

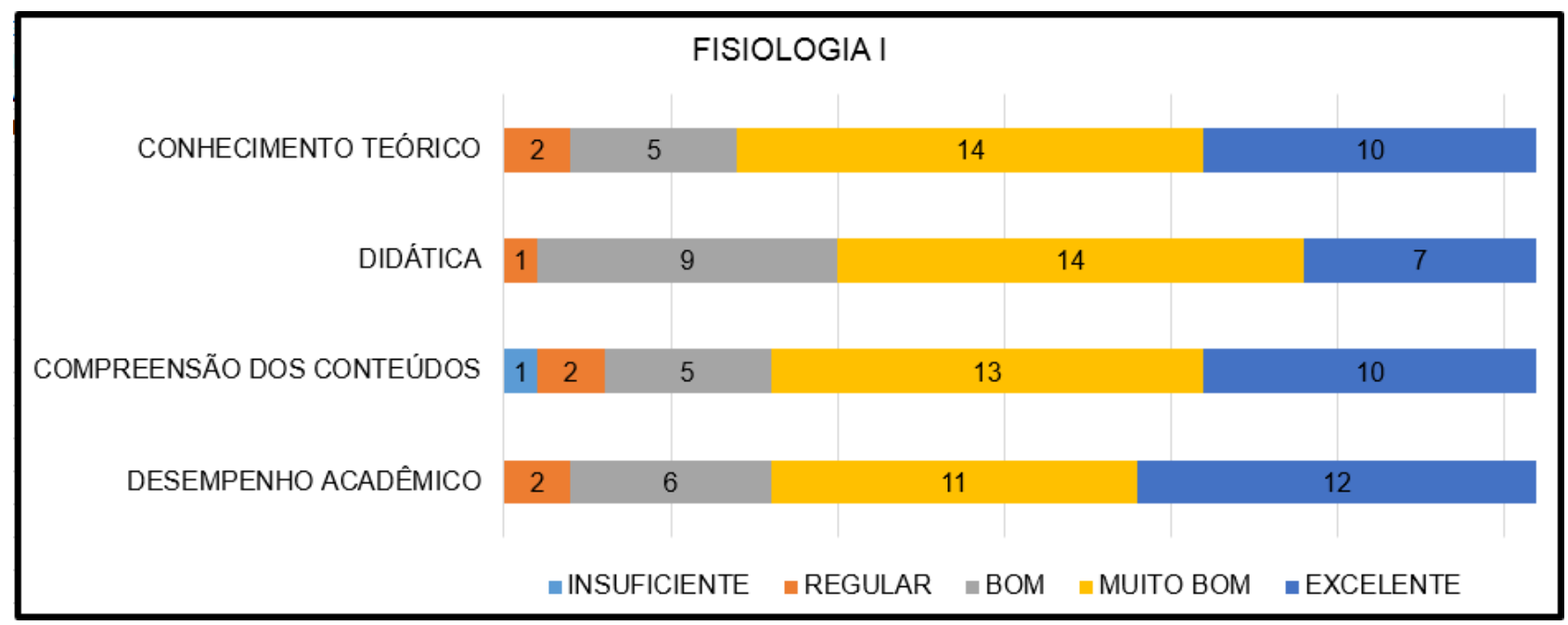

Figura 5. Percepção dos estudantes de Fisiologia I sobre as atividades de monitorias, dados apresentados em frequência absoluta.

Já, dentre os alunos que cursaram a disciplina de Fisiologia II, 90,6\% (39 alunos) responderam ao questionário por terem participado das atividades de monitoria. Novamente foi observada a maior frequência para as classificações "Muito Bom" e "Excelente" em todos os quesitos (Figura 6).

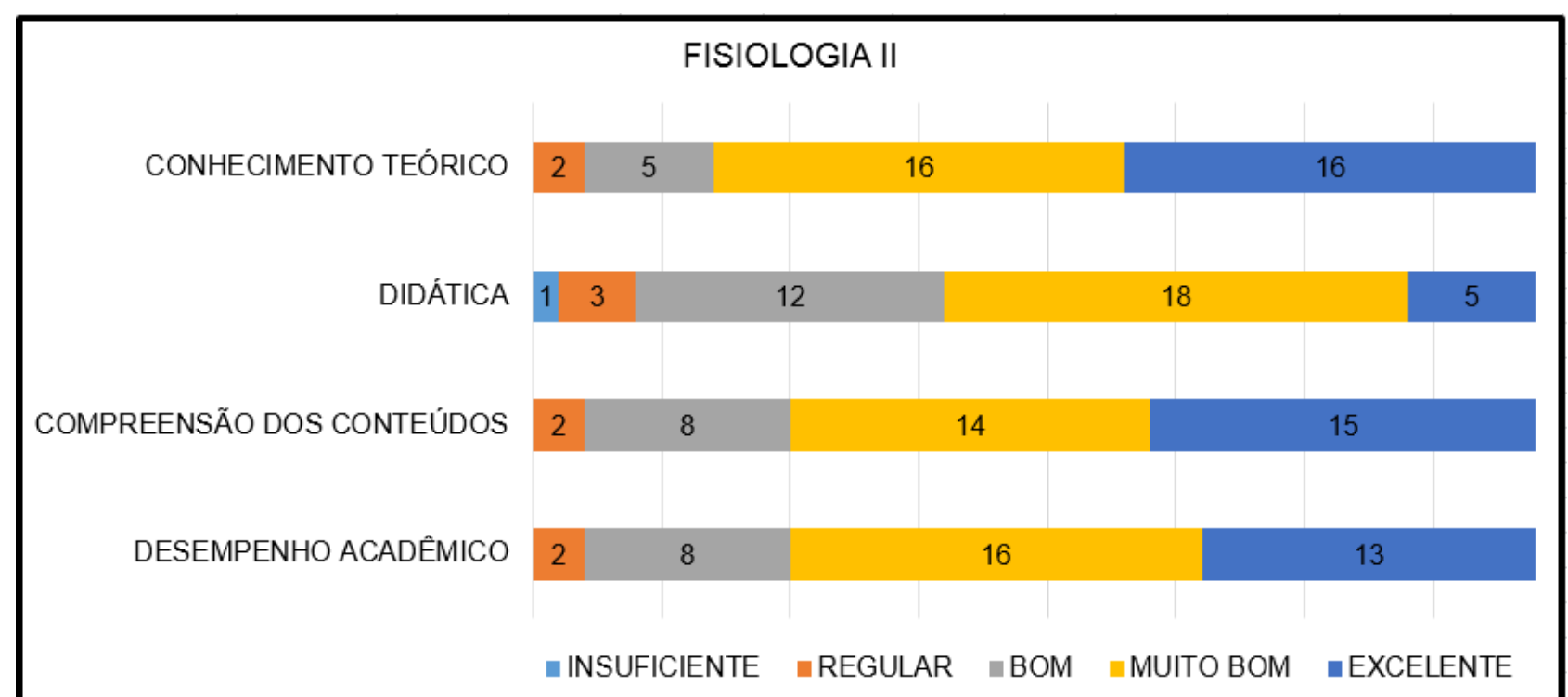

Figura 6. Percepção dos estudantes de Fisiologia II sobre as atividades de monitorias, dados apresentados em frequência absoluta.

A última pergunta deste eixo do questionário foi destinada aos alunos que não participaram das atividades de monitoria. Os dados apontaram os seguintes motivos pelos quais isso ocorreu: falta de tempo, falta de interesse e melhor aprendizado estudando sozinho.

Além das atividades de monitoria, os integrantes da Liga realizaram a apresentação de casos clínicos durante as aulas de discussões de questões práticas, 
Pesquisa em ensino: Academic League of Medical Physiology: a view of the students about active teachinglearning methodologies

com a supervisão dos professores, tendo como objetivo relacionar os conhecimentos básicos do primeiro ano com as matérias posteriores do curso. 31,1\% dos alunos da Fisiologia I classificaram a atividade como "Boa", sendo que 28,8\% afirmaram que essa atividade foi "Muito Boa" e 22,2\% "Excelente". Já, dentre os alunos que cursaram Fisiologia II, 41,8\% classificaram como "Muito Boa" e 25,5\% como "Excelente".

O desenvolvimento dos casos clínicos com os discentes baseou-se principalmente na ideia de flexibilidade cognitiva, que é a capacidade de assimilar informações e conceitos para solucionar problemas. Sua avaliação positiva corrobora com a literatura, uma vez que essa habilidade melhora o processo de ensino-aprendizagem de áreas como fisiologia e medicina, nas quais muitos conceitos são utilizados simultaneamente para a resolução de questões complexas. Os discentes precisam desenvolver essa capacidade, pois processos rígidos, mecânicos e passivos são insuficientes, enquanto a elaboração cognitiva e a exercitação constroem uma base de conhecimento mais sólida [4, 6, 30].

Um estudo realizado em Salvador também utilizou a escala Likert para avaliar a percepção de alunos de dois cursos de medicina sobre o uso de metodologias ativas no ensino de fisiologia. Foi evidenciada uma avaliação bastante positiva dessas práticas e, além disso, apesar de não ter ocorrido consenso em relação à substituição completa do modelo passivo por metodologias ativas, com respostas tendendo ao centro na escala Likert (3 pontos), demonstrou-se a potencialidade do uso deste método, que pode ser integrado e não necessariamente excludente ao modelo tradicional expositivo de ensino [9]. Outros trabalhos também demonstram que o uso de metodologias ativas é aprovado tanto por docentes quanto por discentes e que pode ser integrado ao currículo tradicional $[3,4,6,7]$.

Os resultados obtidos neste estudo sugerem que o avanço de apenas um semestre no curso permite um melhor aproveitamento das formas integrativas de ensino que relacionam as disciplinas básicas com as mais avançadas. O ensino baseado na problematização dos conteúdos objetiva colocar o estudante como sujeito ativo nos cenários educacionais, permitindo reconhecer as individualidades da prática, as demandas e os cuidados no cotidiano dos serviços de saúde [31]. A fisiologia humana é uma ciência básica e essencial para a compreensão dos demais componentes curriculares, assim, as práticas que incentivem o aluno a buscar um estudo continuado, aprofundado e atualizado nesta área, relacionando-o à realidade concreta de atuação do graduando, se tornam benéficas para a formação acadêmica [32]. 
Pesquisa em ensino: Academic League of Medical Physiology: a view of the students about active teachinglearning methodologies

Há que se apontar que, dependendo de sua configuração, esses grupos de atividades extracurriculares podem trazer tanto benefícios quanto malefícios, isso porque as LAs não devem objetivar tão somente o suprimento de deficiências curriculares dos acadêmicos, mas sim gerar cenários nos quais se exerçam o ensino e a aplicação prática $[33,34]$. O fenômeno do aumento do número de LAs nas mais diversas instituições do país não foi acompanhado de uma reflexão sobre seu papel dentro das instituições, ou mesmo de sua atuação como grupo educativo, por esse motivo a avaliação da sua função para formação do participante precisa ser ampliada [35]. Esse controle é necessário, pois, independente das metas, as LAs são um instrumento que dá voz e protagonismo aos discentes acerca de seu aprendizado [36].

Dessa forma, percebe-se o impacto positivo que as atividades do projeto de ensino Liga Acadêmica de Fisiologia Médica podem trazer para a formação de seus participantes e dos alunos monitorados. Trata-se de um processo formativo fundamental, já que acontecem várias atividades de reflexões coletivas e se articulam conteúdos conceituais à análise de práticas médicas futuras. É nesse espaço que surge uma nova atuação, que deve ser acompanhada de avaliações permanentes para constante reconstrução dos seus objetivos, além de propiciar uma troca contínua entre discentes e docentes, possibilitando o protagonismo e a identificação com o meio acadêmico, como local de vivências, experiências e pertencimentos [20].

\section{Considerações Finais}

A Liga Acadêmica de Fisiologia Médica possibilita o desenvolvimento de habilidades de comunicação, de apresentação de seminários e de leituras críticas de artigos. Assim, os monitores afirmaram desenvolver uma autonomia maior, aumentar seu senso de responsabilidade e melhorar o vínculo com professores, colegas e alunos monitorados. Dessa forma, para além do ganho intelectual, sugerimos que há uma melhora na relação interpessoal entre os sujeitos e crescimento mútuo de habilidades entre monitores e monitorados.

A maioria dos acadêmicos considerou que os monitores estão preparados para desenvolver as atividades, ademais, os acadêmicos afirmaram melhorar sua compreensão acerca dos conteúdos e o seu desempenho acadêmico quando participam das monitorias.

A principal diferença encontrada se deu em relação às apresentações de casos clínicos durante as aulas: os alunos que estavam cursando Fisiologia II tiveram uma 
Pesquisa em ensino: Academic League of Medical Physiology: a view of the students about active teachinglearning methodologies

percepção mais positiva da atividade quando comparados àqueles que estavam cursando Fisiologia I. Tal resultado sugere que o avanço de cada semestre no curso faz com que a compreensão consiga ser mais ampla, aumentando a capacidade e o interesse dos alunos em participar e compreender atividades que estimulam um raciocínio mais voltado à prática profissional.

Além disso, embora o presente trabalho não objetivasse a formulação de diretrizes que norteassem a criação e o funcionamento das LAs, especificamente da LAFIM, com avaliações continuadas, fomentou a discussão sobre as práticas desse grupo e demonstrou que processos internos de autoavaliação podem ser criados a fim de monitorar e aprimorar suas práticas.

Por fim, observou-se que o projeto de ensino da Liga Acadêmica de Fisiologia Médica é um método ativo complementar de ensino-aprendizagem, que objetiva promover uma discussão continuada, capacitando alunos a auxiliar estudantes de diferentes semestres no estudo biológico. Métodos de ensino como esse proposto são cada vez mais comuns no ambiente da universidade, nos quais os estudantes deixam de ser espectadores e se tornam protagonistas do processo de aprendizagem, no entanto, ainda são poucos seus processos de monitorização e de avaliação, que devem ser amplamente debatidos, a fim de não transformar esses grupos em meros apêndices curriculares que cumprem funções paliativas de suprir as carências do ensino universitário.

\section{Referências}

[1] Frison LMB. Monitoria: uma modalidade de ensino que potencializa a aprendizagem colaborativa e autorregulada. Pro-Posições. 2016; 1(27),133-153. DOI: 10.1590/0103-7307201607908

[2] Santos MP. O Papel do Ensino Superior na proposta de uma educação inclusive. Revista Movimento. 2003; (7), 78-91. Available from: http://www.lapeade.com.br/publicacoes/artigos/Paper\%20UFF.pdf

[3] Anbarasi M, Rajkumar G, Krishnakumar S, Rajendran P, Venkatesan R, Dinesh T, Mohan J, Venkidusamy $\mathrm{S}$. Learning style-based teaching harvests a superior comprehension of respiratory physiology. Advances in Physiology Education. 2015; 39 (3), 214-217. DOI: 10.1152/advan.00157.2014

[4] Tsang A, Harris DM. Faculty and second-year medical student perceptions of active learning in an integrated curriculum. Advances in Physiology Education. 2016; 40 (4), 446-453. DOI: 10.1152/advan.00079.2016

[5] LaDage LD, Tornello SL, Vallejera JM, Baker EE, Yan Y, Chowdhury A. Variation in behavioral engagement during an active learning activity leads to differential knowledge gains in college students. Advances in Physiology Education. 2018; 42 (1), 99-103. DOI: 10.1152/advan.00150.2017

[6] Miller CJ, Metz MJ. A comparison of professional-level faculty and student perceptions of active learning: its current use, effectiveness, and barriers. Advances in Physiology Education. 2014; 38 (3), 246-252. DOI: 10.1152/advan.00014.2014

[7] Vázquez-García M. Collaborative-group testing improves learning and knowledge retention of human 
Pesquisa em ensino: Academic League of Medical Physiology: a view of the students about active teachinglearning methodologies

physiology topics in second-year medical students. Advances in Physiology Education. 2018; 42 (2), 232239. DOI: $10.1152 /$ advan.00113.2017

[8] Borges S, Mello-Carpes PB. Undergraduate students as promoters of science dissemination: a strategy to increase students' interest in physiology. Advances in Physiology Education. 2015; 39(1),133-136. DOI: 10.1152/advan.00120.2014

[9] Quintanilha LF, Costa GN, Coutinho MR. Medical student perceptions about active methodologies in the study of physiology in medical schools in Salvador, Brazil. Advances in Physiology Education. 2018; 42 (4), 693-696. DOI: 10.1152/advan.00105.2018

[10] Freeman S, Eddy SL, McDonough M, Smith MK, Okoroafor N, Jordt H, Wenderoth MP. Active learning increases student performance in science, engineering, and mathematics. Proceedings of the National Academy of Sciences of the United States of America. 2014; 111(23), 8410-8415. DOI: 10.1073/pnas.1319030111

[11] Altermann CDC, Neves BHS, Mello-Carpes PB. The inclusion of undergraduate students in physiology outreach activities improves their physiology learning and understanding skills. Advances in Physiology Education. 2016; 40(1), 529-532. DOI: 10.1152/advan.00012.2016

[12] Altermann CDC, Garcia A, Mello-Carpes PB. Apresentação de resultados de pesquisa científica como estratégia para aumentar o interesse dos alunos em fisiologia. Journal of Biochemistry Education. 2016; 14(1), 89-98. DOI: 10.16923/reb.v14i1.562

[13] Souza CS, Iglesias AG, Pazin-Filho A.. Estratégias inovadoras para métodos de ensino tradicionais aspectos adicionais. Revista da Faculdade de Medicina de Ribeirão Preto. 2014. 47(3), 284-92. DOI: 10.11606/issn.2176-7262.v47i3p284-292

[14] Lara MV, Borges S, Welter M, Mello-Carpes PB. Objetos de aprendizagem como coadjuvantes do processo de ensino-aprendizagem de Fisiologia humana. Revista de Ensino em Bioquímica. 2014. DOI: 10.16923/reb.v12i1.273

[15] Monteiro LLF, Cunha MS, Oliveira WL, Bandeira NG, Menezes JV. Ligas Acadêmicas: o que há de positivo? Experiência de implantação da Liga de Cirurgia Plástica. Revista Brasileira de Cirurgia Plástica. 2008; 23(3),158-61. Available from: http://www.rbcp.org.br/details/405/pt-BR/ligas-academicas--o-que-ha-depositivo--experiencia-de-implantacao-da-liga-baiana-de-cirurgia-plastica

[16] Gonçalves RJ, Ferreira EAL, Gonçalves GG, Lima MCP, Cerqueira ATARC, Correa FK, Smaira SI, Torres AR. Quem "Liga" para o Psiquismo na Escola Médica? A Experiência da Liga de Saúde Mental da FMB - Unesp. Revista Brasileira de Educação Médica. 2009; 33 (2), 298-306. DOI:10.1590/S0100$\underline{55022009000200019}$

[17] Gonçalves R, Lara MVS, Altermann CDC, Mello-Carpes PB. Using discussion forums on topics of general interest as a strategy to improve the students' interest in Physiology. Journal of Biochemistry Education. 2017; 15(1), 6-14. DOI: 10.16923/reb.v15i1.680

[18] Carroll RG, Silverthorn DU, Wehrwein EA. Undergraduate and Medical School Physiology Education in the United States. Physiology. 2017; 32(1), 262-263. DOI: 10.1152/physiol.00012.2017

[19] Costa BEP, Hentschke MR, Silva ACC, Barros A, Salerno M, Figueiredo CEP, Antonello IC, Lopes MHI. Reflexões sobre a importância do currículo informal do estudante de medicina. Revista Scientia Medica. 2012; 22(3), 162-168. Available from: http://revistaseletronicas.pucrs.br/ojs/index.php/scientiamedica/article/ download/10052/8190

[20] Queiroz SJ, Azevedo RLO, Lima KP, Lemes MMDD, Andrade M. A Importância das Ligas Acadêmicas na Formação Profissional e Promoção de Saúde. Fragmentos de Cultura. 2014; 24, 73-78. Available from: http://seer.pucgoias.edu.br/index.php/fragmentos/article/viewFile/3635/2125

[21] Ferreira DAV, Aranha RN, Souza MHFO. Ligas Acadêmicas: uma proposta discente para ensino, pesquisa e extensão. Interagir: pensando a extensão. 2011; (16), 47-51. Available from: https://www.epublicacoes.uerj.br/index.php/interagir/article/view/5334/3934 
Pesquisa em ensino: Academic League of Medical Physiology: a view of the students about active teachinglearning methodologies

[22] Lins LF, Ferreira LMC, Ferraz LV, Carvalho SSG. A importância da monitoria na formação acadêmica do monitor. Anais IX Jornada de Ensino, Pesquisa e Extensão. 2009; Available from: http://www.eventosufrpe.com.br/jepex2009/cd/resumos/r0147-1.pdf

[23] Likert R. A technique for the measurement of atitudes. Archives in Psychology. 1932; 140, 1-55.

[24] Schleich ALR, Polydoro SAJ; Santos AAA. Escala de satisfação com a experiência acadêmica de estudantes do Ensino Superior. Avaliação Psicológica. 2006; 5(1), 11-20. Available from: http://pepsic.bvsalud.org/pdf/avp/v5n1/v5n1a03.pdf

[25] Dalmoro M, Vieira KM. Dilemas na Construção de Escalas tipo Likert: o número de itens e a disposição influenciam nos resultados?. Revista Gestão Organizacional. 2013, 6, edição especial, 161-174. Available from: http://bell.unochapeco.edu.br/revistas/index.php/rgo/article/viewFile/1386/1184

[26] Neves BHS, Ferreira IS, Machado RS, Mello-Carpes PB. "Physiologying" study group using active methods: the student's perception about the use os this strategy in human Physiology teaching-learning. Journal of Biochemistry Education. 2018; 16(1), 74-84. DOI: 10.0.66.27/reb.v16i1.794

[27] Machado RS, Mello-Carpes PB. Status of research on physiology education in Brazil. Advances in Physiology Education. 2018; 42 (4), 547-554. DOI: 10.1152/advan.00036.2018

[28] Barbosa MG, Azevedo MEO, Oliveira MCA. Contribuições da monitoria acadêmica para o processo de formação inicial docente de licenciandas do Curso de Ciências Biológicas da FACEDI/UECE. Revista de Ensino de Biologia da Associação Brasileira de Ensino de Biologia (SBEnBio). 2014; (7), 5471-5479. Available from: https://docplayer.com.br/46769876-Contribuicoes-da-monitoria-academica-para-o-processode-formacao-inicial-docente-de-licenciandas-do-curso-de-ciencias-biologicas-da-facedi-uece.html

[29] Silva DT, Martins TL, Santos AV, Goulart NS, Silva JA, Souza DC. A Evolução do processo ensinoaprendizagem através da implantação da liga acadêmica. Revista Rede de Cuidados em Saúde. 2014; 8 (2), 1-5. Available from: http://publicacoes.unigranrio.edu.br/index.php/rcs/article/view/2277/1131

[30] Rhodes AE, Rozell TG. Cognitive flexibility and undergraduate physiology students: increasing advances knowledge acquisition within an ill-structured domain. Advances in Physiology Education. 2017; 41 (3), 375382. DOI: $10.1152 / a d v a n .00119 .2016$

[31] Gomes MPC, Ribeiro VMB, Monteiro DM, Leher EMT, Louzada RCR. O uso de metodologias ativas no ensino de graduação nas Ciências Sociais e da Saúde - Avaliação dos Estudantes. Ciência e Educação. 2010; 16(1), 181-198. DOI: 10.1590/S1516-73132010000100011

[32] Hamamoto Filho PT. Ligas Acadêmicas: Motivações e Críticas a Propósito de um Repensar Necessário. Revista Brasileira de Educação Médica. 2011; 35(4), 535-543. DOI: 10.1590/S0100-55022011000400013

[33] Hamamoto Filho PT, Villas-Bôas PJF, Corrêa FG, Muñoz GOC, Zaba M, Venditti VC, Schellini SA. Normatização de abertura de ligas acadêmicas: a experiência da Faculdade de Medicina de Botucatu. Revista Brasileira de Educação Médica. 2010; 34 (1), 160-167. DOI: 10.1590/S0100-55022010000100019

[34] Bonin JE, Oliveira JGS, Nascimento JM, Rezende ME, Stopato SP, Leite ICG. Liga Acadêmica de medicina de família e comunidade: instrumento de complementação curricular. Revista de APS. 2011; 14 (1), 50-57. Available from: http://ojs2.ufjf.emnuvens.com.br/aps/article/view/14623/7836

[35] Torres AR, Oliveira GM, Yamamoto FM, Lima MCP. Academic Leagues and medical education: contribuitions and challenges. Interface - Comunicação, Saúde, Educação. 2008; 12 (27), 713-20. DOI: $\underline{10.1590 / S 1414-32832008000400003}$

[36] Santana ACDA. Ligas acadêmicas estudantis: o mérito e a realidade. Medicina. 2012; 45 (1), 96-8. Available from: http://revista.fmrp.usp.br/2012/vol45n1/PV_Ligas\%20Acad\%EAmicas\%20Estudantis.pdf 